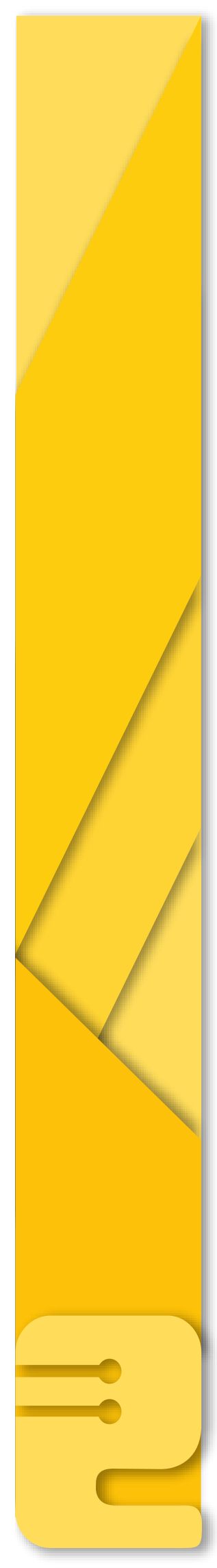

\title{
CARACTERIZACIÓN FÍSICO-MECÁNICA DE LAS FIBRAS DE LA PALMA DE CHONTADURO
}

Nelson Vargas Ortiz ${ }^{1}$

Fecha de recibido: Julio 23 de 2015 | Fecha de aprobado: Septiembre 16 de 2015

\section{Resumen}

Con el objetivo de adaptarse a la demanda de una sociedad de consumo cada vez mayor y a la necesidad de generar un menor impacto ambiental, se ha producido un gran interés en el área de investigación direccionada a la búsqueda de materiales procedentes de fuentes renovables, que en muchos casos son subutilizados. Por ello, en el presente estudio se investiga sobre las fibras de la palma de chontaduro procedentes de la ciudad de Buenaventura en la región pacifica de Colombia; esto con el objetivo de determinar si las fibras del material pueden ser utilizadas y tener aplicaciones en el área de construcción civil. En la primera fase se han realizado estudios de caracterización empleando técnicas de microscopía óptica (MO) y microscopía electrónica de barrido (MEB) así como ensayos de resistencias mecánica, mediante los cuales fue posible determinar una alta resistencia de las fibras obtenidas de la palma de chontaduro.

Palabras clave: palma de chontaduro, pruebas físico-mecánicas, estructura de materiales compuestos, materiales y tecnologías no convencionales.

\footnotetext{
1 Pontificia Universidade Catolica Do Rio De Janeiro
} 


\title{
PHYSICO- MECHANICAL CHARACTERIZATION OF THE FIBERS OF THE PALM OF CHONTADURO
}

\begin{abstract}
With the objective of adapting to the demand of a consumer society that's continually growing and the need of generating a lesser environmental impact, a big interest has been produced in the researching field that aims to search for materials coming from renewable sources, which are misused in many cases. Because of this, the present study researches about the peach palm's fibers coming from the city of Buenaventura from the pacific region of Colombia; this with the objective of determining if such fibers can be used and have applications in the field of civil construction. In the first stage, studies of characterization have been realized employing techniques of optical microscopy and scanning electronic microscopy as well as mechanical strength trials, through which it was possible to determine a high resistance of the obtained fibers from the peach palm tree.
\end{abstract}

Keywords: peach palm tree, physical-mechanical trials, composed materials' structure, nonconventional materials and technologies.

\section{CARACTERIZAÇÃO FÍSICO-MECÂNICA DAS FIBRAS DA “PALMEIRA DE PUPUNHA”}

\section{Resumo}

com o objetivo de adaptar-se à alta demanda de uma sociedade de consumo cada vez maior, e à necessidade de gerar menos impacto ambiental, há um grande interesse na pesquisa de materiais procedentes de fontes renováveis, que em muitos casos são subutilizados. Desta forma, pesquisam-se as fibras da palmeira da "Pupunha" procedentes da cidade de Buenaventura, na região pacífica da Colômbia, isto com o objetivo de determinar se as fibras do material podem ser utilizadas e aplicadas na área da construção civil. Na primeira fase, realizaram-se estudos de caracterização, empregando técnicas de microscopia óptica (MO) e microscopia eletrônica de varredura (MEB), bem como ensaios de resistência mecânica, mediante os quais foi possível determinar uma alta resistência das fibras obtidas da palmeira de "Pupunha".

Palavras-chave: palma de pupunha; provas físico-mecânicas, estrutura de materiais compostos; materiais e tecnologias não convencionais. 


\section{INTRODUCCIÓN}

La palma de chontaduro (Bactris gasipaes) es nativa de la región amazónica, de la cual se extrae sus frutos y palmitos para el consumo. La explotación de esta palma es muy reciente debido a la implementación del agro negocio; pero la cultura del chontaduro es vieja en la región tropical, y su domesticación tiene orígenes antiguos, cuando los nativos comenzaron las actividades agrícolas.

La producción de palmitos a nivel mundial va en aumento; por ejemplo, en Brasil, actualmente, se consumen 100 toneladas por año. El chontaduro está reemplazando el uso de palmas como açai, juçara y otras palmas nativas por ser más productiva en rendimiento y crecimiento.

En la actualidad los productores de palmitos dedican grandes áreas para la plantación, con miras a la producción de frutos y semillas. Cuando esta palma tiene 12 años de edad se corta para darle paso a las palmas emergentes, al realizar este proceso se generan grandes cantidades de tallos y fibras, los cuales poseen unas características similares a la madera de especies aprovechables.

La planta se adapta a una amplia gama de condiciones ecológicas en las zonas tropicales. Asimismo, es originaria de las áreas tropicales con alta precipitación y suelos pobres, crece mejor cuando la lluvia es abundante y se puede cultivar desde el nivel del mar hasta los 800 metros de altitud.

El tallo de la palma se utiliza frecuentemente en la elaboración de artesanías o como combustible para la generación de energía, algunas comunidades lo utilizan como material de construcción con buenas propiedades tanto mecánicas como de resistencia a la intemperie.

Las fibras naturales son materiales que diariamente se desechan. Éstas al poseer propiedades mecánicas interesantes y un bajo costo, se convierten en un material atractivo para la industria. Del mismo modo, debido a la facilidad de obtención y procesamiento, éstas se pueden utilizar principalmente como refuerzo de materiales compuestos para aumentar la rigidez y resistencia.

Para la realización de refuerzos con fibras naturales es necesario caracterizar el material y determinar las propiedades mecánicas, para esto es necesario realizar tanto estudios de microscopia óptica (MO) y microscopia electrónica de barrido (MEB) para determinar la estructura y microestructura como ensayos de tracción para determinar la resistencia del material analizado.

Figura 1. Palma de chontaduro obtenidas en la zona de Buenaventura.

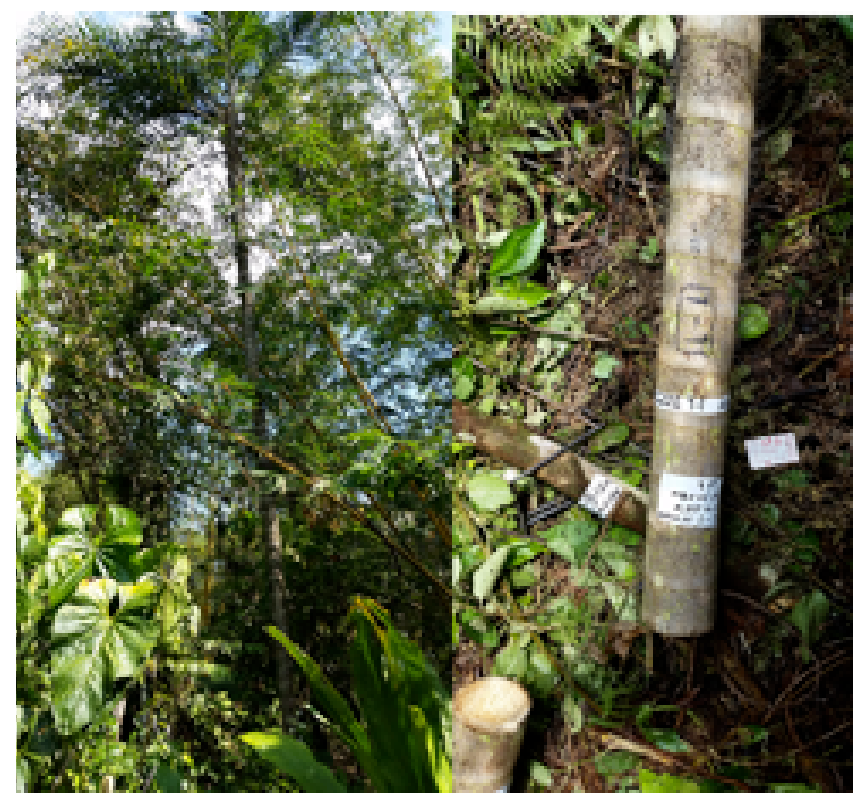

Fuente: Elaboración propia 
En este orden de ideas, el objetivo de este artículo es estudiar las propiedades físico-mecánicas de las fibras de la palma de chontaduro, para determinar la viabilidad en la utilización como material alternativo de construcción en obras civiles.

La palma de chontaduro empleada en este estudio es originaria de Buenaventura, Valle del Cauca, especialmente en un suelo arcillo-arenoso con bajos niveles de fertilidad y un alto nivel de humedad.

\section{RESULTADOS Y ANÁLISIS}

Se obtuvieron muestras de palma con una longitud promedio de 12 metros y con edad aproximada de 8 años, con buenas características de uniformidad de diámetro y sin ningún tipo de ataque por roedores o insectos (ver figura 1).

Cuando son realizados cortes transversales a la estirpe de la palma con una edad mayor a 8 años, se observa una alta resistencia de la pared externa de la misma y está compuesta principalmente por fibras largas alineadas axialmente y con propiedades similares a especies arbóreas. La macro-estructura de la misma es comparable con la capa externa del bambú, por tal motivo se realizó el análisis estructural a las palmas de chontaduro utilizando la siguiente metodología:

Para la obtención de las imágenes por MO, fueron producidos cuerpos de prueba en formas de pequeños bloques al realizar cortes precisos sobre la pared de la palma (ver figura 2), que posteriormente fueron pulidos en una maquina rotativa intercambiando lijas desde la número 200 a 1000 . La muestra fue girada $90^{\circ}$ para generar una superficie uniforme y finalmente se pasó por una pulidora de paño rotativa con la adición de pasta de diamante de 20 y 10 micrones.

Figura 2. Corte transversal de la palma de chontaduro y pared externa de la misma.

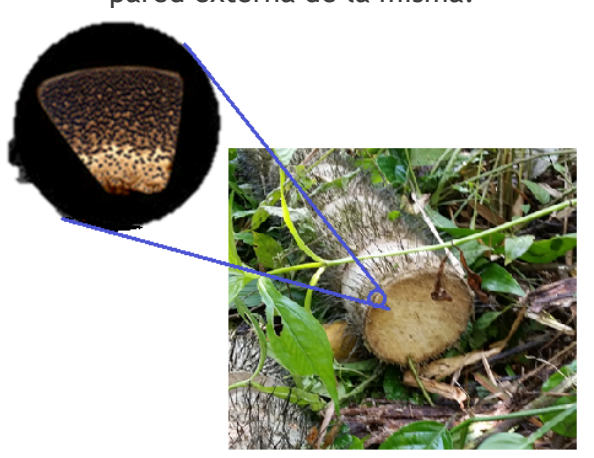

Fuente: Elaboración propia
Los análisis por MEB y ensayos de tracción fueron realizados empleando fibras obtenidas manualmente de las palmas de chontaduro. Para tal efecto fue necesario la utilización de técnicas manuales de aplastado de las mismas con herramientas de percusión que evitan golpear las bordas para no dañar las fibras. Por otro lado, la extracción individual de las fibras es realizada con ayuda de un cepillo de cerdas metálicas. Este proceso es realizado cuando la palma ha perdido el $90 \%$ del contenido de humedad, aspecto que favorece el desprendimiento de las fibras con la matriz. Las fibras obtenidas están en un rango de $10 \mathrm{a}$ $50 \mathrm{~cm}$ de largo, como se puede observar en la figura 3 .

Figura 3. Fibras secas obtenidas de la palma de chontaduro luego del proceso de extracción.

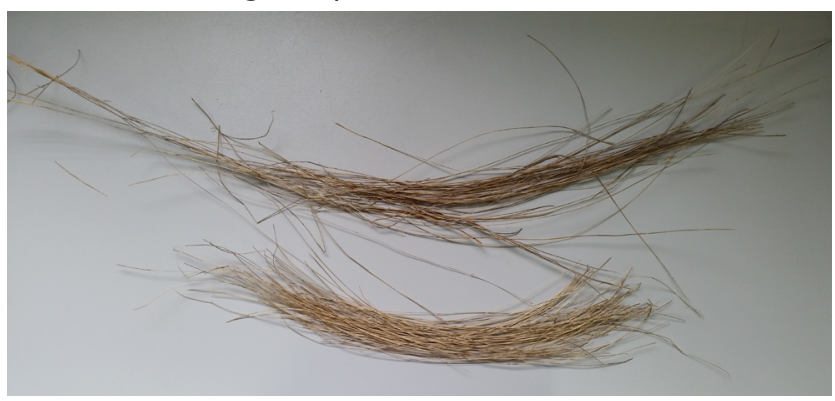

Fuente: Elaboración propia

Las imágenes obtenidas por MO de una sección de la pared interna de la palma de chontaduro permiten la determinación de una distribución no homogénea de fibras (ver figura 4), así como la identificación de grietas en algunas de las fibras presentes en la muestra. Al realizar un análisis detallado, se observa que existen variaciones en la estructura de la pared de la palma, especialmente en la concentración y tamaño de las fibras, que presentan un gradiente descendente de concentración en dirección al eje central del estipe (ver figura 2). También, fue realizada la relación entre fibras y ductos (ver tabla 1), mediante la ayuda del software de dominio público Fiji sobre la imagen representada en la figura 5 ( $a$ y b).

Figura 4. Imagen de MO del cuerpo de prueba.

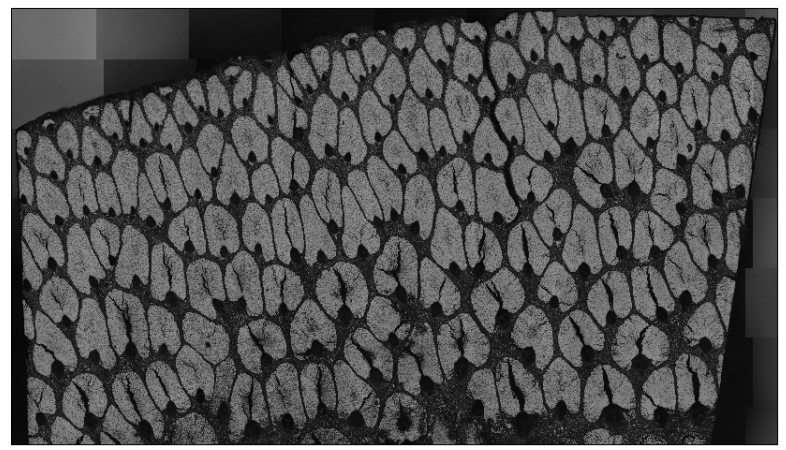


Tabla 1. Análisis de partículas con Fiji.

\begin{tabular}{|l|c|c|c|c|}
\hline & \multicolumn{2}{c|}{ NÚMERO } & \multicolumn{2}{c|}{ ESTADO DE DUCTOS } \\
\cline { 2 - 5 } & Fibras & Ductos & Buenos & Damnificados \\
\hline Real & 172 & 163 & & $34 \%$ \\
\hline Sin plugin & 188 & 195 & $64 \%$ & $36 \%$ \\
\hline Con plugin & 169 & 182 & $71 \%$ & $29 \%$ \\
\hline
\end{tabular}

Fuente: elaboración propia

Mediante la caracterización por MEB fue posible la determinación de la estructura interna de fibras individuales, mostrando estar compuesta principalmente de ductos en variedad de tamaño como se observa en la figura 6. Por otro lado, mediante la utilización del software Fiji se determinó el área transversal de las fibras en 0,2 $\mathrm{mm}^{2}$ (ver figura 6).

Figura 5. Caracterización de las imágenes obtenidas por MO de las fibras mediante FIJI.

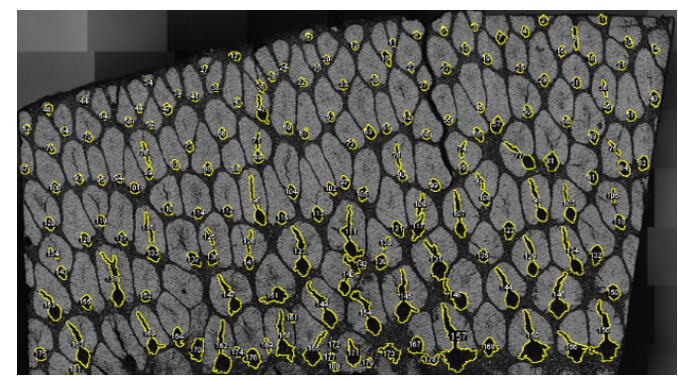

(a)

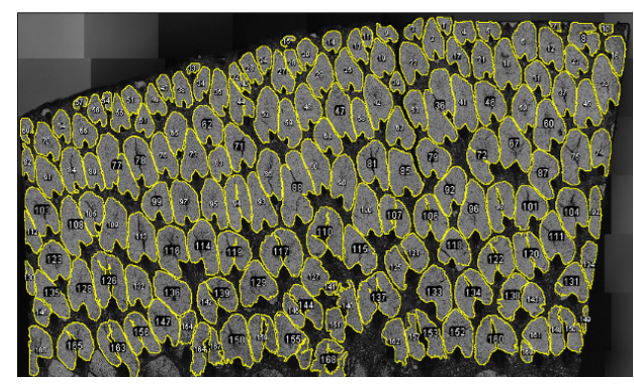

(b)

Fuente: Elaboración propia
Figura 6. Imagen de MEB de una fibra de la palma de chontaduro.

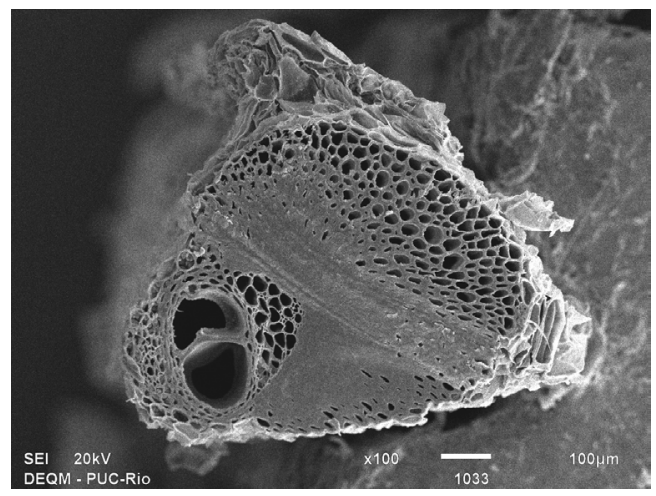

Fuente: Elaboración propia

Los ensayos de tracción fueron realizados bajo la norma ASTM D-3379. Para tal efecto, fueron preparados cuerpos de prueba con fibras individuales reforzadas en papel cartón para concentrar la carga de tracción uniformemente sobre las fibras (ver figura 7). Los ensayos de tracción fueron realizados en un equipo de prueba de accionamiento mecánico equipado con mordazas de acción neumática. La velocidad de la prueba utilizada fue $2 \mathrm{~mm} / \mathrm{min}$ y fueron probadas 15 muestras con un promedio de longitud de $30 \mathrm{~mm}$.

Figura 7. Cuerpo de prueba de una fibra de la palma de chontaduro empleado en el ensayo de tracción.

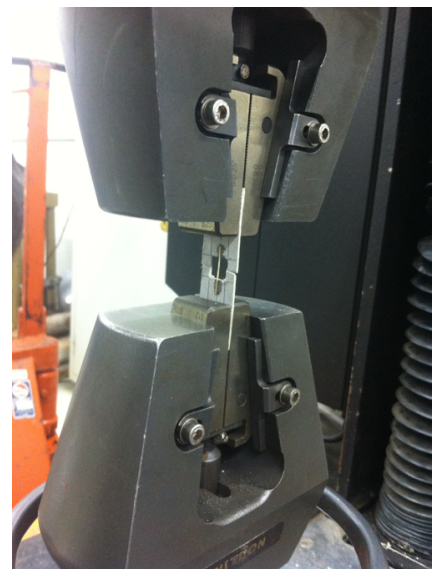

Fuente: Elaboración propia 
Figura 8. Resultados obtenidos del ensayo de tracción reali-

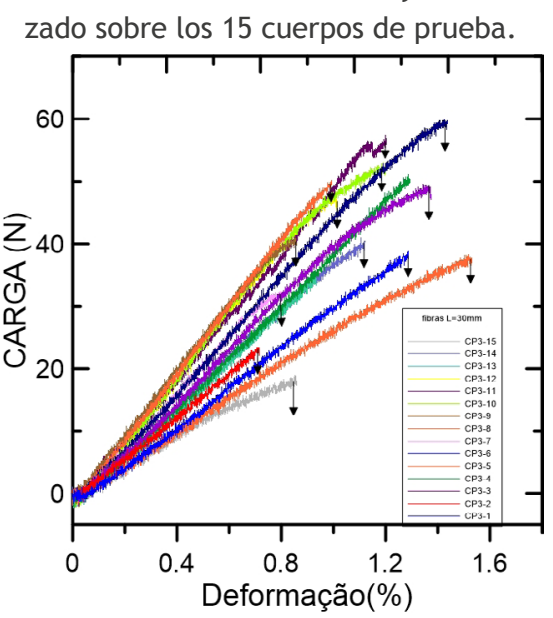

En la figura 8 se muestran los resultados obtenidos en los ensayos de tracción realizados a las fibras de palma de Chontaduro. Al analizar los resultados de la carga soportada por las fibras hasta la fractura y al dividir por el área de las fibras, se obtiene una resistencia media de $200 \mathrm{MPa}$. De esta forma, estos materiales presentan una resistencia aceptable y las fibras podrían ser usadas como refuerzo de materiales compuestos y empleados en la construcción de obras civiles.

\section{CONCLUSIONES Y RECOMENDACIONES}

Los resultados experimentales obtenidos muestran que las fibras se pueden obtener fácilmente de la palma con la ayuda de elementos convencionales y con el uso de equipos de bajo costo.

A partir de los resultados experimentales se podría concluir que las fibras de chontaduro tienen un gran potencial para ser utilizado como refuerzo de materiales compuestos de matriz polimérica y cementicia.

Las fibras de la palma de chontaduro al ser obtenidas de los residuos sobrantes de la producción de las plantaciones de la misma, ofrecen un valor agregado a la agroindustria en el reaprovechamiento de residuos.
Al realizar el análisis de los resultados se recomienda para investigaciones futuras estudiar fibras de la palma de chontaduro de diferentes zonas tropicales, por cuanto se ha demostrado cambios en sus las propiedades físico-mecánicas en estudios realizados con palmas de Brasil y Colombia.

\section{REFERENCIAS}

1. Temer, B., D `Almeida J. (2008). Estudo das Propriedades físico mecânicas do material lenhoso da palmeira Pupunha. Rio de Jainero: PUC-RIO.

2. Villate. J. (2015). Disertaçao de maestrado en desarrollo,. Rio de Jainero: PUC-RIO.

3. Tamayo, G. (2010). Investigación Estudio del chontaduro, análisis de Sus propiedades, explotación Aplicación creativa y en el ámbito culinario. Tesis de grado. Universidad Tecnológica Equinoccial, Quito, Ecuador.

4. Wegst, U. (2011). Doblado eficiencia a través de gradientes de propiedad en bambú, palma y materiales compuestos a base de madera. En: revista del comportamiento mecánico de materiales biomédicos, 4: 744-755.

5. Martínez-Eraso, D. (2009). Valores característicos determinación de la resistencia a la torsión y tensión en Guadua angustifolia Kunth. Tesis de Grado. Universidad Nacional de Colombia, Bogotá, Colombia.

6. Encuentra, N. (2011). Bambú: materiales de alta tecnología para refuerzo de concreto. Tesis doctoral. Pontificia Universidad Católica de Río de Janeiro, Rio de Janeiro. Brasil. 